\title{
Driver Assistance System based on Raspberry Pi
}

\author{
Narayan Pandharinath Pawar \\ Student \\ Sinhgad Academy of Engineering \\ Pune, Maharastra, India
}

\author{
Minakshee M.Patil \\ Asst. Professor \\ Sinhgad Academy of Engineering \\ Pune, Maharastra, India
}

\begin{abstract}
Automotive Electronics sector having more demand due to day by day use of embedded system for different applications in car. Most of luxurious cars having more automatic controls like Airbags,ABS,ESP,ECU,ESP,climate control \& more. Automatic Guided Vehicle (AGV) nothing but vehicle guideline provided by capturing images of the road. Intelligent driver assistance system nothing but provide the full assistance to the driver when drive driving the car on the road along with considering the traffic intensity \& white lane detection. System consist of the camera module used to take continuous video streaming. This stream video store into SD card first $\&$ process this video by writing the script in python. Lane detection is done from the video by using the Hough Transform Algorithm \& Hough lines. Design the system in such a way that by considering small Robot as the demo module \& one is pilot car it acts as obstacle in all the direction. Detection of the obstacle in front $\&$ rear direction means it need different type of obstacle detector sensors to detect the obstacle. Ultrasonic sensor it acts as the obstacle detector to detect the any obstacle within the range of 4 meters. Full driver assistance provided by detecting the side lane by taking the video streaming by using camera mounted on the car \& obstacle detection is done by using the Ultrasonic sensor module. It is possible to display the distance apart from obstacle in meter on the display. Display consist of TFT screen connected to the system to display the continuous video \& distance from other car. All system implemented on the new platform Raspberry Pi Development Board having ARM1176-JFZS core \& BCM Audio-Video Codec with operating frequency $700 \mathrm{MHz}$.Board support TFT screen as well as the HDMI support[6]. Vehicles need to be readvancing by video transmission among vehicles for safety and cooperative deriving. The video images captured from camera could help the driver to monitor the surroundings as well as transmit the compressed images over vehicular communication network Video over wireless communication has a lot of potential applications in intelligent transportation systems (ITS). Capturing the video is done by taking the continuous Video of the road lanes.Video streaming utilizes high bandwidth data links to transmit information. The highbandwidth systems required larger equipment, better line-ofsight, and more complex mechanism for reliable transmit ion over the network. The intended platform for the system described in this study, is to develop a software defined algorithm for automatic video compression and transmission. The proposed algorithm is able to robustly find the left and right boundary of the lane using Hough Transform method and transmit over the network. Therefore the limitations of high-bandwidth equipment become more significant in a tactical scenario. The results show that the proposed method works well on marked roads and transmission in various lighting conditions[7].
\end{abstract}

\section{General Terms}

Driver Assistance provide by detecting the side lanes of the road when car moving. Hough Transform \& Hough lines used to detect the white lanes from the video streaming. Obstacle detection done by using Ultrasonic sensor. Edge detection, Histograms equalization et. al.

\section{Keywords}

ARM1176- core,Raspberry Pi,Robot,USB webcam,Ultrasonic

\section{INTRODUCTION}

Driver Assistance system nothing but provide the assistance for the driver when he driving the car. It includes the lane departure warning system by detecting the white lanes of the road. Reverse parking assistance provided by using Ultrasonic Sensor by sending the trigger \& wait for echo response. This system based on Raspberry Pi with having ARM11 \& BCM Audio-Video codec which need to used Raspbean operating system. By using the ultrasonic sensor on front ,back. Hough transform \& Hough line is used to detect the side lanes. This transform algorithm is used to process the continuous video streaming \& process video display on the TFT screen. It is possible to turn on the camera by writing the script in python for GPIO's \& USB 2.0 Webcam. Driver assistance nothing but detecting the side lane means to provide lane departure warning system by using Hough Transform algorithm with obstacle detecting warning using Ultrasonic sensors. Continuous video process is done by Detecting the white lines using Hough lines.This Driver assistance nothing but to provide the driver assistance by detecting white lanes .Using the Hough transform \& Hough lines it is possible to process the continuous video getting from the webcam. Ultrasonic sensor module is used to detect the obstacle. This system is completely based on the Raspberry Pi with having ARM11 core with BCM audio-video codec from Broadcom which need TFT screen to display the camera video on the screen. If driver driving the car in the reverse direction so ultrasonic sensor used to detect the obstacles. Captured image is bulky $\&$ robust difficult to process \& storage[1].Reverse control the car by detecting the obstacle using ultrasonic sensors it is possible to measure the distance between the two cars by calculating the response time getting time from the system it need to calculate the distance \& display on the screen. It is possible to Design the GUI using the python. There are many application examples including multiple trailer trucks, articulated buses, boat or camping trailers, airport luggage carriers and passenger vehicles. The fabrication cost of passive trailers is lower than active vehicles. The operation cost is much lower because the [2] system used to provide the assistance for the driver.

\section{STRUCTURE AND PRINCIPL}

System consist of the 2 ultrasonic sensor it is used for detecting the obstacles in front $\&$ rear direction with distance in meter in range. camera used for taking the front video. DC 
Motor driver L293D used to drive the DC motors \& also used for to increase the current rating for DC motors. DC motors used to give rotation to the wheels. It can be forward \& reverse direction.USB 2.0 webcam is used to take the continuous video. These video processes by using the Hough transform \& display process video on the TFT screen. Raspberry Pi Development board is used to control all system with it having Raspbean Linux operating system with having Linux kernel. It is possible to run project to create the GUI interface using python to provide the user interface with the system. Figure 1.shows the actual implementation of the system by considering the all parameters. Ultrasonic sensor is acts as RADAR used to detect the obstacle. This system implemented on the one Robot module. Robot module on the

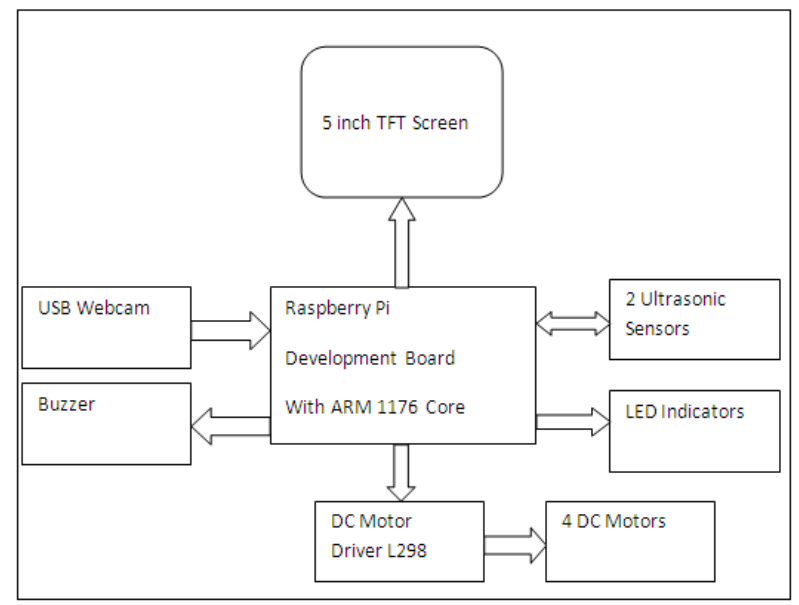

Fig 1:Block Diagram of the System

system nothing but connect the four DC motors with wheels to the system it acts as the 4 wheels of the car. DC motor driver L293D used to drive the DC motor. One 12V DC battery mounted Robot to provide supply to system. Buzzer is used to give the warnning to the driver if driver changing the lane. Also LED indicator gives the indication about the changing the lane. If lane not change the Green LED turns ON. If lane change the Red LED turns ON.

\section{HARDWARE DESIGN}

\subsection{Ultrasonic Sensor Modules}

Ultrasonic module is used to detect the obstacles in all side. It having 4 meter range. it used to detect the obstacles very precise manner.

\subsection{USB 2.0 Webcam Camera Module}

It is used to take the continuous video in front as well as back side with 30 frames per second. It is used for just capturing the images from the outside the world.

\subsection{Motor Driver L293D}

It is used to drive the DC motor in all the direction control through the program, it is also possible to change the power supply polarity from programming. It having the $600 \mathrm{~mA}$ current rating.

\subsection{Motors 300 rpm}

In car module DC Motor is used to drive the wheels connected to the car module. Also it is possible to control the speed of the DC motor by using L293D.

\subsection{TFT LCD Screen}

It is used to provide the complete user interface with the system. It is also used to show the distance apart from the other car in all the directions. Also it shows the front $\&$ rear side video on the screen as per user changing the direction or gear in front $\&$ back direction.

\subsection{Raspberry Pi Development Board}

It is the main heart of the system it is used to control all the system. It having ARM 11 Core with BCM Audio-Video Codec used to provide the GPIO's with having operating frequency $700 \mathrm{MHz}$.

\subsection{Buzzer}

It is used to siren the when lane changes by the driver means it assist the driver $\&$ gives warning about the lane change.

\subsection{Indicator}

It is used to giver proper indication to the driver about correct $\&$ safty driving into the proper lane. If correct lane then Green LED turns ON \& if lane change occurs the it gives the Red LED turns ON.

\section{SOFTWARE DESIGN}

\subsection{Porting Raspbean OS}

To port Linux kernel on the Raspberry Pi board take 4/8GB SD card place Raspbean OS kernel image file in card make it bootable.

\subsection{Installation Of Tool chain}

Python programming it need to install packages of the python

for writing script in python it need to install python in Raspberry Pi, for Image capturing \& video streaming it needs other libraries by using following commands we can install

\$sudo apt-get install python

\$sudo apt-get install ffmpeg

\$sudo apt-get install fswebcam

\$sudo apt-get install omxplayer

\$sudo apt-get install gstreamer-0.10

after installing this we can take image by writing

\$fswebcam -r 1280x720 cam1.jpg

\$gstreamer-0.10 used by using gst-launch-0.10 plugin

\$gst-launch-0.10 videotestsrc ! ffmpegcolorspace ! autovideosink

$\$$ gst-launch $=0.10$ videotestsrc $!$ pattern $=11$ ! ffmpeggcolorspace ! autovideosink

then write the script for webcam for accessing the camera video \& GPIO's to control the DC motors ,Ultrasonic Sensors, Buzzer \& LED by importing the BCM GPIO's. 


\subsection{Flowchart -Design flow of System}

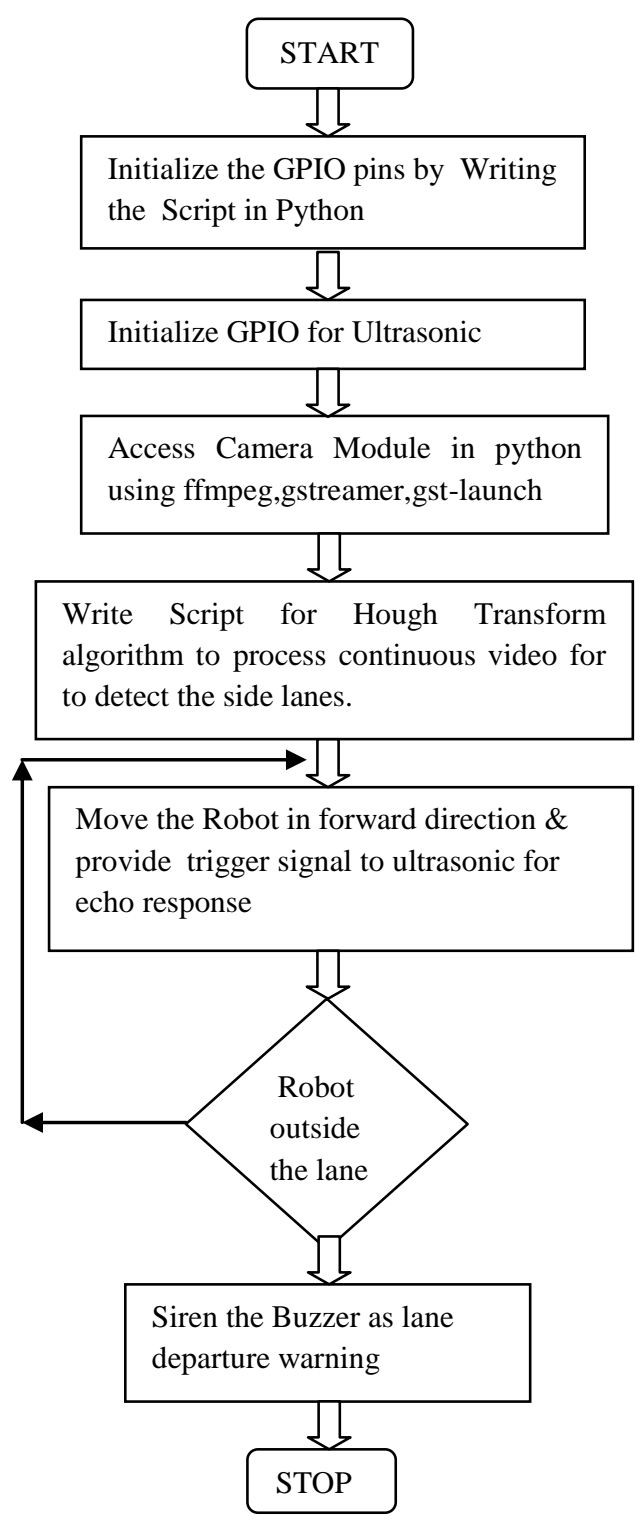

Fig 2: Design Flow Diagram of System

\section{IMPLEMENTATION}

\subsection{Raspberry Pi Development Board}

Raspberry Pi consist of having ARM1176EJ-FZS core with BCM Audio-video codec with having SPI,I2C Facilities with number of GPIO,700MHz speed.

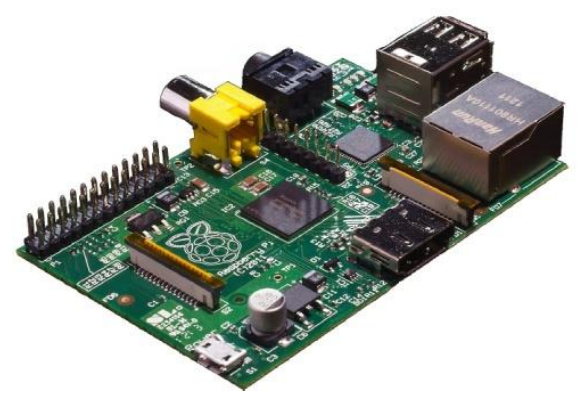

Fig 3: System Development Board

\subsection{Demo Model}

Demo module consist of the actual the Raspberry Pi board with TFT screen connected to the all the peripherals with having the ultrasonic interface, Dc motor interface, camera interface, Battery interface with 4 wheel chasse system it like the on car module of the system .

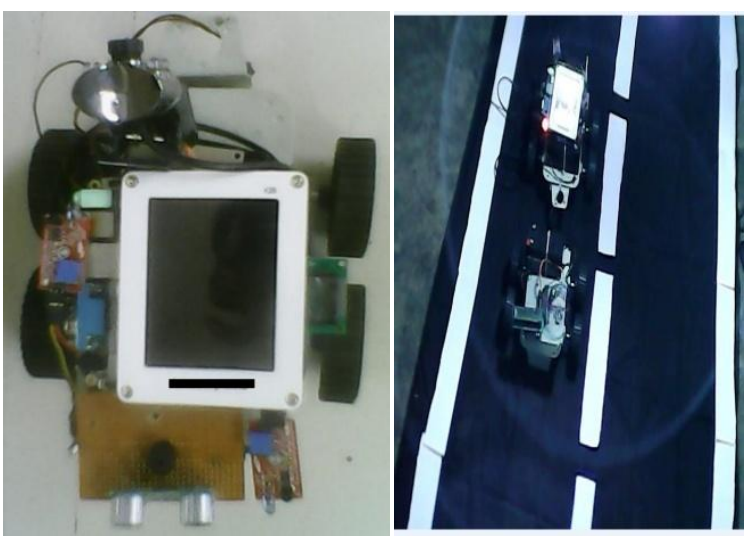

Fig 4: Demo Module

It having onboard peripherals like Ethernet port USB 2.0 port,3 UART, GPIO pins, TFT screen interface connector, also it having onboard Raspbean operating system within it having Boot mode \& NVROM flash mode also. It supports all higher level languages like $\mathrm{C}, \mathrm{C}++$ also ,so it is possible to write device driver program to control the speed of the DC motors as well as to control the Ultrasonic sensor modules \& also implemented the TFT screen driver it is used to show all the parameters on the screen. So writing python script we can interface GPIO pins by importing library files of the raspberry pi development board for example test.py we can write this script in shell of the Raspbery python editor IDLE or directly on the

import RPi.GPIO as gpio import time

gpio.setmode(gpio.BCM)

gpio.setup(25, gpio.OUT)

while True:

gpio.output(25, gpio.HIGH)

time.sleep $(1)$

gpio.output(25, gpio.LOW)

time.sleep $(1)$

after this we can run by pressing $\mathrm{Ctrl}+\mathrm{S} \&$ then run it by pressing F5.Then for capturing video streaming it need to write another script. We can run this on command prompt

\$python test.py

For continuous video streaming avconv or ffplay

\$avconv -f video4linux2 -I /dev/video0 video0.avi

\$ffplay -f video4linux2 -framerate -video_size 320x240 /dev/vodeo

If we want to see output on HDMI the use

\$omxplayer -o hdmi myvideo.mp4

To show capture image use

\$fbi cam.jpg

In this system design in such a way that it is used to provide the complete driver assistance about the car safety. Ultrasonic 
sensor it work on the principle of the RADAR it sends first the trigger signal at that time system starts the timer \& when echo receive from the system the timer stop at that time we are getting the time by using this time it is possible to measure the distance of the other car.Lane departure warning given by detecting the white lines of the road when actually car moving on road.Camera connected to Raspberry Pi board it takes the inages \& send to TFT screen for display purpose. Hough transform is used to detect the lane \& display on to the TFT screen.Hough Transform algorithm is used to process the video stream getting from the camera. In Hough transform consist of the Hough line used to detect the white lines of the when moving car moving on the road. If robot on the lane then buzzer siren means system gives warning to the driver about lane departure.So,it is nothing but the lane departure system of the car.

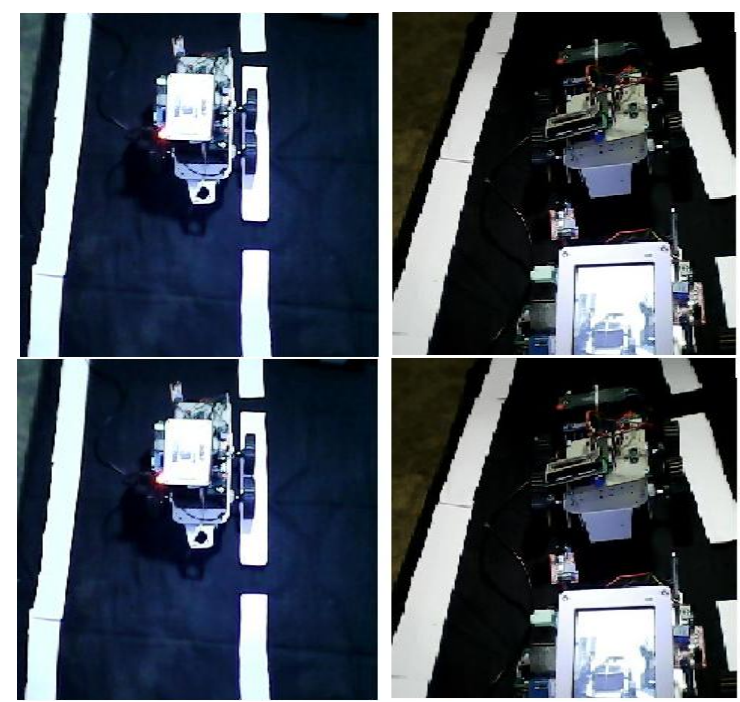

Fig5: Case Study Module

Design consideration case study demo module consist of one Robot Module consist of one webcam, Ultrasonic sensors, DC motors, Raspberry Pi development board along with TFT screen. Also for demo purpose Fig-5: show that the actual consider of the road lane module made on the chart sheet paper.

\section{RESULTS}
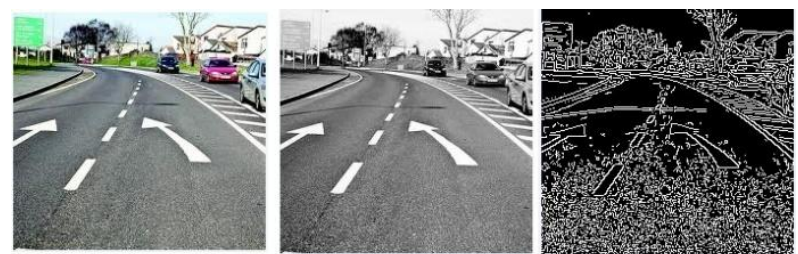

Fig6:a)RGB Image b)Gray Image c)Hough Transformed
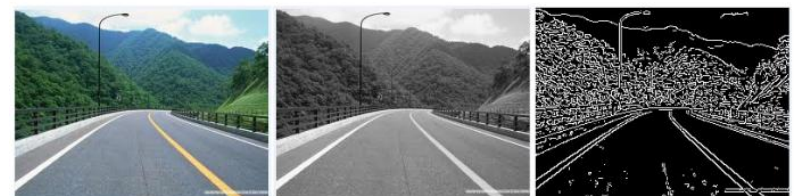

Fig7 :a)RGB Image b)Gray Image c)Hough Transformed

\section{CONCLUSION}

We have purpose this system to provide the complete driver assistance about each \& every movement of the car in particular direction. System based on the ARM Samsung platform so the operating frequency of this board is very high it fulfill the requirement of the system. The System is implement for to provide the complete driver assistance when driver driving the car on the road to avoid the damage of the car. This part of the car system implemented by observing the scenario of the day today life. Hough lines is very sufficient to detect the white lanes of the road \& provide the driver assistance system by giving the lane departure warning.

\section{ACKNOWLEDGEMENT}

I would like to thanks my friend Mr.Hrushikesh Takalkar from Tata Honeywell,Pune,Mr.Heramb Joshi from Softdel System,Pune \& Mr.Shivam Khole from Sphier Unified Solution Pvt.Ltd,Pune who gives more contribution to implementing this system based on the Automotive Embedded System Design \& processing domain.

\section{REFERENCES}

[1] Cheng Yao-yu Liu Yong -lin,Liang Ying,Wang Shiqin,'Design of image acquisition \& storage system Based on ARM \& Embedded",ICEOE,IEEE2011.

[2] Jae Il Roh, Hyunsuk Lee, Woojin Chung," Control of a Car with a Trailer using the Driver Assistance System", International Conference on Robotics and Biomimetics,IEEE2011.

[3] A. Jazayeri, H. Cai, J. Y. Zheng,"Motion Based Vehicle Identification in Car Video", Intelligent Vehicles Symposium University of California, San Diego, CA, USA,IEEE 2010

[4] Sunil Acharya, Colin Tracey, Abbas Rafii Canesta," System Design of Time-of-Flight Range Camera for Car Park Assist and Backup Application".

[5] Chan-Hong Chao, Cheng-Hsiang Ho, Sheng-Hsiung Lin and Tzuu Hseng S. Li," Omni-Directional VisionBased Parallel-Parking Control Design for CarLikeMobile Robot".

[6] Narayan.P.Pawar,MinaksheeM.Patil,'Intellignt Diver Assistance System for Automotive",IJRET2014.

[7] Shabana HABIB, Mahammad A Hannanl," Lane Departure Detection and Transmisson using HoughTransform Method", ,"”, PRZEGLĄD ELEKTROTECHNICZNY, ISSN 0033-2097, R. 89 NR $5 / 2013$

[8] Saravanan Kannan, Arunkumar Thangavelu, RameshBabu Kalivaradhan," An Intelligent Driver Assistance System (I-DAS)for Vehicle Safety Modelling using OntologyApproach", International Journal Of UbiComp (IJU), Vol.1, No.3, July 2010. 